\title{
Preliminary results of conformal radiation therapy for medulloblastoma
}

\author{
Thomas E. Merchant, ${ }^{1}$ Laura Happersett, Jonathan L. Finlay, and Steven A. Leibel \\ Department of Radiation Oncology, St. Jude Children's Research Hospital, Memphis, TN 38105-2794 [T.E.M.]; \\ New York University Medical Center, Hassenfeld Children's Center for Cancer and Blood Disorders, New York, \\ NY 10016 [J.L.F.]; and Memorial Sloan-Kettering Cancer Center, New York, NY 10021 [L.H., S.A.L.]
}

Radiation therapy for medulloblastoma consists of postoperative irradiation of the intracranial and spinal subarachnoid volume with an additional boost to the primary site of disease in the posterior fossa. The entire posterior fossa is usually included in the boost volume. Conformal radiation therapy techniques may be used to boost the primary site alone and substantially reduce the dose received by normal tissues, including the supratentorial brain, the middle and inner ear, and the hypothalamus. Using these techniques to irradiate only the tumor bed or residual tumor and not the entire posterior fossa represents a new paradigm in the treatment of medulloblastoma. In this study, we examine the use of conformal radiation therapy in the treatment of 14 patients with medulloblastoma. These patients were treated with multiple static, individually shaped, noncoplanar beams directed at the primary site after craniospinal irradiation. Excluding two patients who had previously received irradiation to the posterior fossa, the mean dose delivered to the primary site was $5715 \mathrm{cGy}$. Among the medulloblastoma patients $(n=10)$ who received immediate postoperative radiation therapy, no failures have occurred with a median follow-up of 42 months (range from 30 to 54 months). To demonstrate the differences in the distribution of dose to normal tissues when comparing conventional and conformal techniques, dose-volume histograms

Received 23 March 1998, accepted 1 February 1999.

${ }^{1}$ Address correspondence and reprint requests to Thomas E. Merchant, DO, PhD, Department of Radiation Oncology, St. Jude Children's Research Hospital, 332 N. Lauderdale, Memphis, TN 38105-2794.

${ }^{2}$ Abbreviations used are as follows: CRT, conformal radiation therapy; CTV, clinical target volume; DVH, dose-volume histogram; GTV, gross tumor volume; PTV, planning target volume.

${ }^{3}$ Aggarwal, R., Thompson, S.J., McHaney, V.A., and Kun, L.E. (1996) Unpublished data. of the total brain, middle and inner ear, hypothalamus, and temporal lobe were created and presented for an example case. The neurologic, neuroendocrine, and neurocognitive outcome for patients with medulloblastoma may be influenced with the use of conformal radiation therapy. The use of these techniques should be formally tested in prospective studies of rigorously staged patients with failure rate monitoring. Neuro-Oncology 1, 177-187, 1999 (Posted to Neuro-Oncology [serial online], Doc. 9801, June 15, 1999. URL <neuro-oncology.mc.duke.edu>)

$T$ he advent of $\mathrm{CRT}^{2}$ planning and delivery has invited a reassessment of the principles and practices commonly employed for the treatment of certain posterior fossa tumors including medulloblastoma, ependymoma, and cerebellar astrocytoma. CRT requires the delineation of anatomic compartments, normal tissue structures, eloquent or critical areas, and tumor/target volumes using three-dimensional image data. The delineation of specific structures guides the orientation of radiation beams to create isodose surfaces that conform to the target and to avoid or reduce the dose received by normal tissues. One of the objectives of using CRT is to alter the distribution of dose to reduce radiation-related side effects.

When treating medulloblastoma, the posterior fossa has been considered to be a distinct anatomic compartment. This distinction has led to the treatment of this site with opposed-lateral fields using a standard set of guidelines. Exceptions to these rules have been made; however, guidelines, such as those published for most cooperative group studies of medulloblastoma, require treatment of the entire posterior fossa with margins beyond the leptomeninges, anterior brainstem, and tentorium cerebelli. Without modification for differences in tumor location, size, extent of resection, and volume of the residual tumor bed, such treatment may encompass cranial nerves, the middle and inner ear, cervical spinal cord, brainstem, optic chiasm, pituitary gland, hypothalamus, 
Table 1. Clinical and treatment characteristics for 14 patients having medulloblastoma treated with conformal radiation therapy to the posterior fossa

\begin{tabular}{|c|c|c|c|c|c|}
\hline $\begin{array}{l}\text { Patient } \\
\text { age/sex }\end{array}$ & Treatment sequence & Chemotherapy & $\begin{array}{l}\text { Craniospinal } \\
\text { irradiation dose } \\
\text { (cGy) }\end{array}$ & $\begin{array}{l}\text { Posterior } \\
\text { fossa dose } \\
\text { (cGy) }\end{array}$ & $\begin{array}{l}\text { Follow-up } \\
\text { (months) }\end{array}$ \\
\hline$\overline{9 M}$ & GTR-RT/ChemT-ChemT-NED & $\begin{array}{l}\text { Vincristine with RT, then } \\
\text { cisplatin/cyclophosphamide }\end{array}$ & 2,340 & 5,940 & 35 \\
\hline $6 \mathrm{~F}$ & GTR-RT/ChemT-ChemT-NED & $\begin{array}{l}\text { Vincristine with RT, then } \\
\text { cisplatin/cyclophosphamide/vincristine }\end{array}$ & 2,340 & 5,940 & 42 \\
\hline $9 M$ & GTR-RT/ChemT-ChemT-NED & $\begin{array}{l}\text { Vincristine with RT, then } \\
\text { carboplatin/vincristine }\end{array}$ & 3,600 & 5,400 & 54 \\
\hline $21 M$ & GTR-RT-NED & RT alone & 3,600 & 5,940 & 33 \\
\hline $36 M^{b}$ & $\begin{array}{l}\text { GTR-RT-LR-STR-HDCT/ } \\
\text { AuBMT-RT-NED }\end{array}$ & HDCT of cyclophosphamide & 2,400 & 4,500 & 33 \\
\hline $10 M^{b}$ & $\begin{array}{l}\text { GTR-RT-LR-GTR-HDCT/ } \\
\text { AuBMT-RT-NED }\end{array}$ & $\begin{array}{c}\text { HDCT of } \\
\text { carboplatin/thiotepa/etoposide }\end{array}$ & & 4,500 & 32 \\
\hline $26 M$ & STR-RT/ChemT-NED & Vincristine with RT only & 3,600 & 5,400 & 45 \\
\hline $3 F$ & $\begin{array}{l}\text { GTR-ChemT-RT-HDCT/ } \\
\text { AuBMT-RT-POD-DOD }\end{array}$ & $\begin{array}{l}\text { Vincristine/cyclophosphamide/ } \\
\text { cisplatin/etoposide, then } \\
\text { HDCT of carboplatin/thiotepa }\end{array}$ & & 5,940 & \\
\hline $12 M$ & GTR-RT/ChemT-ChemT-NED & $\begin{array}{l}\text { Vincristine with RT, then } \\
\text { cisplatin/cyclophosphamide/vincristine }\end{array}$ & 2,340 & 5,940 & 30 \\
\hline $18 M$ & GTR-RT/ChemT-ChemT-NED & $\begin{array}{l}\text { Vincristine with RT, then } \\
\text { carboplatin/vincristine }\end{array}$ & 3,600 & 5,400 & 47 \\
\hline $32 M$ & GTR-RT-NED & RT alone & 3,600 & 5,940 & 40 \\
\hline $6 M$ & GTR-RT/ChemT-ChemT-NED & $\begin{array}{l}\text { Carboplatin with RT, then } \\
\text { carboplatin/cyclophosphamide }\end{array}$ & 2,340 & 5,400 & 43 \\
\hline $7 F$ & GTR-RT/ChemT-ChemT-NED & $\begin{array}{l}\text { Vincristine with RT, then } \\
\text { cisplatin/cyclophosphamide/vincristine }\end{array}$ & 2,340 & 5,400 & 48 \\
\hline $3 M$ & $\begin{array}{c}\text { STR-ChemT-RT-ChemT-HDCT/ } \\
\text { AuBMT-RT-NED }\end{array}$ & $\begin{array}{l}\text { Cisplatin/cyclophosphamide/ } \\
\text { vincristine/etoposide, } \\
\text { methotrexate/leucovorin, } \\
\text { HDCT of carboplatin/thiotepa. }\end{array}$ & 1,800 & 5,940 & 30 \\
\hline
\end{tabular}

$M$, male; F, female; GTR, gross-total resection; STR, subtotal resection; RT, radiation therapy; ChemT, chemotherapy; NED, no evidence of disease; POD, progression of disease; DOD, dead of disease; LR, local recurrence; HDCT, high-dose chemotherapy; AuBMT, autologous bone marrow rescue.

${ }^{a}$ All patients had no evidence of subarachnoid dissemination.

${ }^{\mathrm{b}}$ Previously received definitive craniospinal irradiation to 3,600 cGy and posterior fossa boost to 5,400 cGy.

supratentorial brain, and noninvolved portions of the cerebellum. Encompassing these additional noninvolved structures engenders many of the late effects that are attributed to radiation therapy. By combining the treatment principles for supratentorial tumors of the same histology with the advantages of CRT, it should be possible to conformally irradiate a well-defined target within the posterior fossa and reduce the dose to normal tissues. To this end we have treated 14 patients with medulloblastoma using these techniques. This report describes our findings based on the experience of planning and delivering treatment to these patients.

\section{Materials and Methods}

\section{Patient Population}

Fourteen patients with medulloblastoma received CRT to their primary site at Memorial Sloan-Kettering Cancer Center between June 1994 and February 1996. Informed consent was obtained in all cases. The following informa- tion was obtained to characterize these patients clinically and according to their treatment: date of birth; sex; date of diagnosis (pathologic); extent of resection; evidence of subarachnoid dissemination; sequencing of surgery, radiation therapy, and chemotherapy; doses and volumes of radiation therapy; radiation therapy treatment planning variables; and chemotherapy agents. The pertinent clinical and treatment related information is presented in Table 1.

\section{Conformal Radiation Therapy: Definition}

External beam radiation therapy may be described as conformal when four criteria are met:

- three-dimensional image data (CT or MRI) are obtained with the patient in the treatment position, and the data are used to reconstruct target volumes and normal tissue structures in three dimensions;

- radiation beams are oriented freely in three dimensions for the planning and delivery process, and structures traversed by the beam are visualized with the eye of the beam;

- the prescription isodose surface matches the PTV; and 
Table 2. Conformal beam orientation for posterior fossa irradiation in 14 cases

\begin{tabular}{|c|c|c|c|c|c|c|c|c|c|c|c|c|c|c|}
\hline & $\mathrm{RL}$ & $\mathrm{LL}$ & RPO & LPO & RAO & LAO & RSO & LSO & SPO & SAO & LAIO & Totals & MLC & Wedge \\
\hline & & & $x$ & $x$ & $x$ & $x$ & $x$ & $x$ & & & & 6 & Yes & 0 \\
\hline & $x$ & $x$ & $x$ & $x$ & & & & & & & & 4 & Yes & 4 \\
\hline & $x$ & $x$ & & & $x$ & & & & & & $x$ & 4 & Yes & 2 \\
\hline & & & $x$ & $x$ & & & & & & $x$ & & 3 & No & 3 \\
\hline & $x$ & $x$ & $x$ & $x$ & & & & & & & & 4 & Yes & 4 \\
\hline & $x$ & $x$ & $x$ & $x$ & & & & & $x$ & & & 5 & Yes & 5 \\
\hline & $x$ & $x$ & $x$ & $x$ & & & & & & & & 4 & Yes & 4 \\
\hline & $x$ & & $x$ & $x$ & & $x$ & & $x$ & & & & 5 & Yes & 5 \\
\hline & $x$ & $x$ & & & & & & & $x$ & & & 3 & No & 2 \\
\hline & $x$ & $x$ & $x$ & $x$ & $x$ & $x$ & & & & & & 6 & Yes & 4 \\
\hline & $x$ & $x$ & $x$ & $x$ & & & & & $x$ & & & 5 & Yes & 4 \\
\hline & $x$ & $x$ & $x$ & $x$ & & & & & & & & 4 & Yes & 2 \\
\hline & $x$ & $x$ & $x$ & $x$ & & & $x$ & $x$ & & & & 6 & No & 4 \\
\hline & $x$ & $x$ & & & $x$ & $x$ & & & & & & 4 & Yes & 4 \\
\hline Totals & 12 & 11 & 11 & 11 & 4 & 4 & 2 & 3 & 3 & 1 & 1 & & & \\
\hline
\end{tabular}

$\mathrm{RL}$, right lateral; LL, left lateral; RPO, right posterior oblique; LPO, left posterior oblique; RAO, right anterior oblique; LAO, left anterior oblique; RSO, right superior oblique; LSO, left superior oblique; SPO, superior posterior oblique; SAO, superior; MLC, multileaf collimator.

- the distribution of dose about the target volume or any structure is computable on a point by point basis in three-dimensional space.

\section{Treatment Planning}

All patients, including those receiving anesthesia for daily treatment, were in the prone position when we planned their treatment and when we treated them, during both the craniospinal and CRT portions of their therapy. Customized immobilization devices that used foam, plastic, or other products were created for all patients. An effort was made to place the patient's head in either a neutral or extended position to minimize the potential for exit dose to the mandible from the posterior spine field. For the CRT portion of the treatment, orthogonal (anterior-posterior and left lateral) radiographs were taken at a distance of $100 \mathrm{~cm}$ from the isocenter of the perceived PTV. This isocenter, considered to be preliminary until verified by its location relative to the center of the contoured PTV, was estimated from pre- and postoperative neuroimaging. A CT scan was performed with the patient in the treatment position; contrast was used in all cases. The gantry of the CT scanner was not angled. During the planning procedure, the isocenter was localized with radiopaque markers on the skin at the locations used to triangulate the patient. The CT field of view was $40 \mathrm{~cm}$, and the scan thickness was $5 \mathrm{~mm}$ for all cases. The entire head and neck regions were imaged. The CT image data were transferred to an off-line treatment planning system.

\section{Conformal Radiation Therapy Planning Methods}

For all cases, the outer contour of the patient's head, brainstem, spinal cord, optic globes, optic nerves, and optic chiasm were contoured. The GTV, which included the postoperative tumor bed and any residual disease, was contoured along with the CTV and PTV. An additional margin of 1.0-2.0 cm was added to the GTV to construct the CTV and an additional margin of $0.5 \mathrm{~cm}$ was added to the CTV to construct the PTV. The CTV represents potential areas of subclinical involvement. The CTV margin was anatomically defined and limited to $1.0 \mathrm{~cm}$ beyond the GTV where anatomic barriers (for example temporal bone, skull, tentorium cerebelli) would limit tumor extension. The margin beyond the GTV was otherwise $2.0 \mathrm{~cm}$. The PTV volume represents the uncertainty in daily reproducibility (ICRU Report 50, 1993). The GTV was contoured with the help of standard pre- and postoperative neuroimaging studies. Once the target volumes and normal structures were contoured, beam orientations were chosen to target the PTV, make the treatment more conformal, and avoid high-dose irradiation of the hypothalamic-pituitary unit, supratentorial brain, and those portions of the temporal bone known to contain the middle and inner ear. The latter was achieved by visualizing the target in the eye of each treatment beam (beam's eye view). Beam's eye view treatment planning is the first step used to select the best beam orientations. The second step is to evaluate the resultant treatment plans and their corresponding DVH data. Each plan was further optimized to achieve homogeneity across the target volume by differentially weighting the individual beams or by adding beam modifiers (wedge filters). Each field was individually shaped and collimated with Cerrobend or a programmed multileaf collimator. Both techniques of collimation were considered to be acceptable. The margin of collimation beyond the PTV was typically $0.5 \mathrm{~cm}$. Patients were treated with 6-MV photons for the craniospinal portion of their treatment (when applicable) or a combination of 6- and 15-MV photons. The number of beams, orientation, type of collimator, and beam modifications are listed in Table 2. The beam orientations used for the patients are graphically depicted in Fig. 1. 


\section{Coronal}

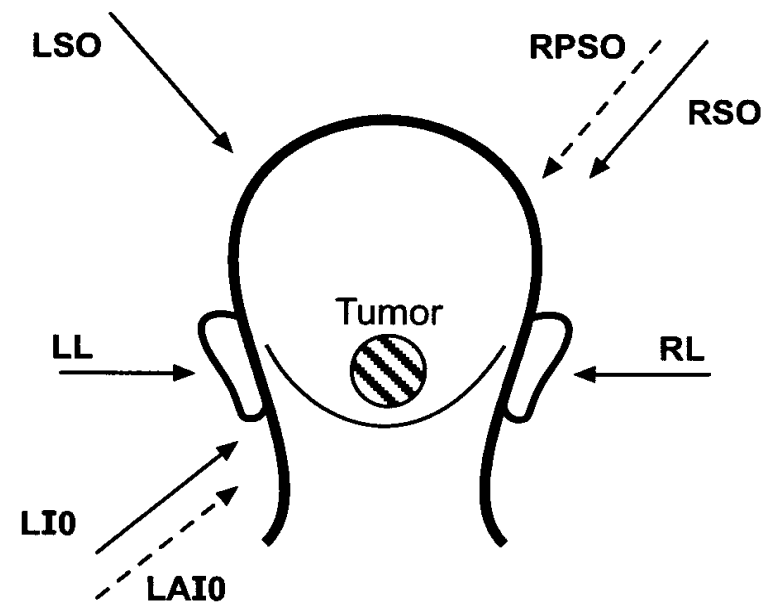

Axial

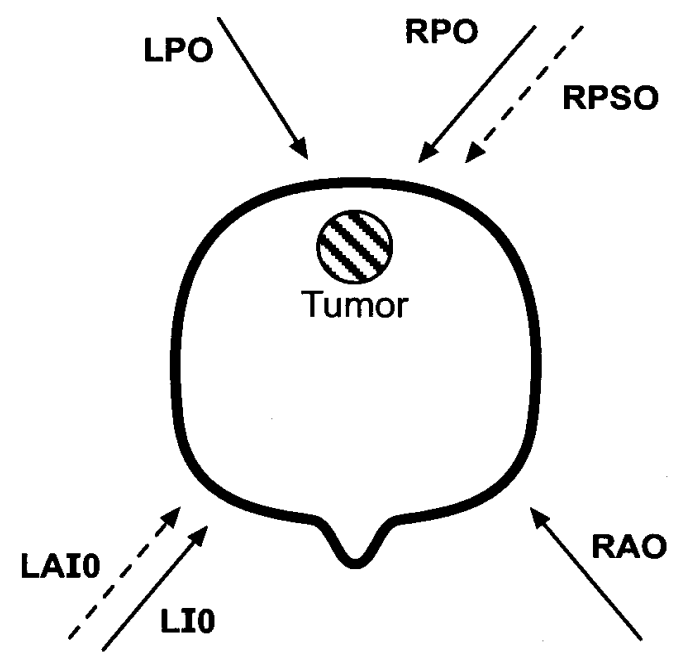

Fig. 1. Beam orientations used to treat the 14 patients with posterior fossa tumors included in this report. $R$, right; $L$, left; $S$, superior; I, inferior; $\mathrm{P}$, posterior; $\mathrm{A}$, anterior; $\mathrm{O}$, oblique; $\mathrm{RL}$, right lateral; $\mathrm{LL}$, left lateral.

\section{Analysis}

One case (of a 7-year-old female) was chosen as an example of the 14 patients treated in this study. This case was chosen because of its symmetrical and relatively spherical tumor bed. It can be described as a completely resected midline posterior fossa tumor that originally filled the IVth ventricle. The contoured GTV was the volume bounded by the dorsal aspect of the brainstem and the resection cavity that was essentially the residual IVth ventricle. Briefly, the GTV in this case did not extend superiorly into the aqueduct of Sylvius, inferiorly into the Foramen of Magendie, laterally into the Foramen of Luschka, or anteriorly to the dorsal aspect of the brainstem. Nine treatment plans were developed to demonstrate the effect of margin and beam orientation on the DVH of specific normal tissue structures, including the entire brain, one of the temporal lobes, one of the auditory structures (middle and inner ear), and the hypothalamus. The nine treatment plans can be described as follows:

2 Field-GTV parallel-opposed left and right lateral portals with a $0.5-\mathrm{cm}$ margin on the GTV

2 Field $-1-\mathrm{cm}$ parallel-opposed left and right lateral portals with a $1.5-\mathrm{cm}$ margin on the GTV

2 Field-2-cm parallel-opposed left and right lateral portals with a $2.5-\mathrm{cm}$ margin on the GTV

Wedge-pair-GTV-paired posterior oblique portals with a $0.5-\mathrm{cm}$ margin on the GTV

Wedge-pair $-1-\mathrm{cm}$ paired posterior oblique portals with a $1.5-\mathrm{cm}$ margin on the GTV

Wedge-pair-2-cm paired posterior oblique portals with a $2.5-\mathrm{cm}$ margin on the GTV
6 Field-GTV 6-field beam arrangement with a $0.5-\mathrm{cm}$ margin on the GTV

6 Field $-1-\mathrm{cm} 6$-field beam arrangement with a $1.5-\mathrm{cm}$ margin on the GTV

6 Field $-2-\mathrm{cm} 6$-field beam arrangement with a $2.5-\mathrm{cm}$ margin on the GTV

All beams used in the example case were 6-MV photons with customized Cerrobend ${ }^{\circledR}$ collimation. Approximately $0.5 \mathrm{~cm}$ of the margin for each case should be considered to be penumbra. The paired posterior oblique portals were beams that were oriented $45^{\circ}$ posteriorly from the lateral position. The six-field beam arrangement was a combination of paired lateral (left and right), posterior oblique (left and right), and superior oblique (left and right) portals. The superior oblique portals were beams that were oriented $45^{\circ}$ superiorly from the lateral position. In addition, we used beam's eye view and a treatment margin of approximately $2 \mathrm{~cm}$ beyond the targeted infratentorial beam to develop a single-treatment plan for irradiating the entire posterior fossa. DVHs for the hypothalamus, total brain, a single temporal lobe, and a single auditory structure were computed for each treatment plan. These data were grouped by structure and plotted to demonstrate the differences in the dose-tonormal-tissue structures as a function of beam arrangement and margin on the GTV. The chosen case represents only one of the many possible scenarios for the GTV. Extension of the tumor toward critical structures will influence the position of the GTV and subsequently the resultant dose-volume histogram. When the GTV includes the Foramen of Luschka, it is likely that the dose to the auditory structures would be increased. Similarly, 
extension of the GTV toward the upper portion of the cerebellum would increase the dose received by the hypothalamus and supratentorial brain, including the temporal lobes. Given the small number of patients, no statistical analysis was applied.

\section{Results}

Table 1 shows demographic and treatment-related information for the 14 patients treated using CRT. The patients included in this report comprised a group of heterogeneously treated patients with medulloblastoma whose ages ranged from 3 to 36 years. None of the patients had evidence of neuraxis dissemination prior to irradiation. Two patients did not receive chemotherapy. Two patients previously received definitive irradiation (5400 cGy) to the posterior fossa. Two of the medulloblastoma patients did not receive craniospinal irradiation in conjunction with CRT to the posterior fossa: One patient was among the two who were previously irradiated, and the other was an infant whom we elected not to treat with craniospinal irradiation. Excluding the patients who were retreated, the mean dose to the posterior fossa primary site was 5715 cGy. Seven of the patients received $5940 \mathrm{cGy}$. For the patients who received radiation therapy as a part of their initial treatment course $(n=10)$, no failures occurred with follow-up ranging from 30 to 54 (median 42) months.

These patients were treated with a variety of coplanar and noncoplanar beam arrangements. The distribution of beam arrangements is outlined in Table 2 along with the type of collimation and beam modifiers. Two patients were treated with three beams, six patients were treated with four beams, three patients were treated with five beams, and three patients were treated with six beams. Beam orientations most commonly used included left and right posterior oblique, left and right lateral, and left and right anterior or superior oblique. The beam arrangements are illustrated in Fig. 1.

One conventional and nine conformal treatment plans were developed from an example case to compare the conformal and conventional methods and to demonstrate the influence of margin and beam orientation on the dose received by normal tissue structures (Figs. $2 \mathrm{~A}-\mathrm{D})$. For each orientation of treatment beams, there is an increase in the integral DVH with increasing margin. The exception is the middle/inner ear with the paired posterior oblique beams. The total brain DVH demonstrates that the paired posterior oblique arrangement may be advantageous over the six-field approach. This indicates that for certain normal tissue structures, alterations in the orientation of the beams, rather than increasing the number of beams, can result in significant gains. The temporal lobe DVH data are similar to those of the total brain. The hypothalamus is spared to a high degree in the plans where the target volume is small. The beam orientations for this demonstration were not specifically chosen to avoid the hypothalamus; therefore, the more complex beam arrangements, such as the paired oblique or sixfield arrangement, may actually increase the dose to this structure for a given margin. The middle and inner ear appear to be spared by the smallest parallel opposed conformal volumes; however, the influence of the position of the GTV, posterior to the dorsal aspect of the brainstem, is largely responsible for this effect.

The DVH data, dose distributions, and target volumes (Figs. 2-4) show that normal tissue sparing is largely an effect of beam orientation and size of the target volume. Beyond these planning parameters, location of the tumor is also a critical factor in normal tissue sparing. While the addition of more beams may increase the conformality of the treatment with respect to the targeted volume, the improvement in conformality may result in the incidental irradiation of normal tissue structures.

\section{Discussion}

\section{Posterior Fossa Irradiation and the Posterior Fossa Boost}

The medulloblastoma patients treated in this study received the boost portion of their treatment to a volume that was smaller than the entire posterior fossa. We assumed that the smaller treatment volume would provide the same probability of disease control as treatment of the entire posterior fossa. Considering the length of follow-up and the lack of failure in the patients treated immediately after their initial resection, it appears that a reduction in the volume of the primary site boost is feasible and potentially as effective as irradiation of the entire posterior fossa. The rationale for treating the entire posterior fossa in a patient with medulloblastoma is not clear. There are conflicting data describing the patterns of local failure for patients with medulloblastoma. The available data challenge the arguments that subarachnoid dissemination at remote locations within posterior fossa may occur with less than full posterior fossa irradiation (Halperin, 1996; Tomita et al., 1986; Wara et al., 1994).

Leptomeningeal failure is a common component of failure that occurs most often in the setting of local failure (Fukunaga-Johnson et al., 1998). Since the purpose of craniospinal irradiation is to treat obvious or subclinical subarachnoid dissemination within and beyond the primary site, inadequate doses in either case should result in failure. What is not clear, however, is exactly how long one must follow a patient who has received insufficient meningeal irradiation before one can demonstrate an excessive rate of failure. In addition, the use of chemotherapy may confound subarachnoid failure analysis. When the neuraxis dose is insufficient, the time to failure should be relatively early compared with time to failure for patients treated with definitive doses. Among 91 patients with low-stage medulloblastoma randomized to 2340 cGy or 3600 cGy neuraxis irradiation, there were excessive early neuraxis failures noted in the 2340 cGy arm with a median follow-up of 16 months (Deutsch et al., 1991). In a recent series of patients treated with definitive irradiation with and without chemotherapy, the median time to failure outside of the primary site was 7 months for patients with M3 disease and 17 months for patients with M1 disease. The median time to any type of failure among patients with disseminated disease was 35 

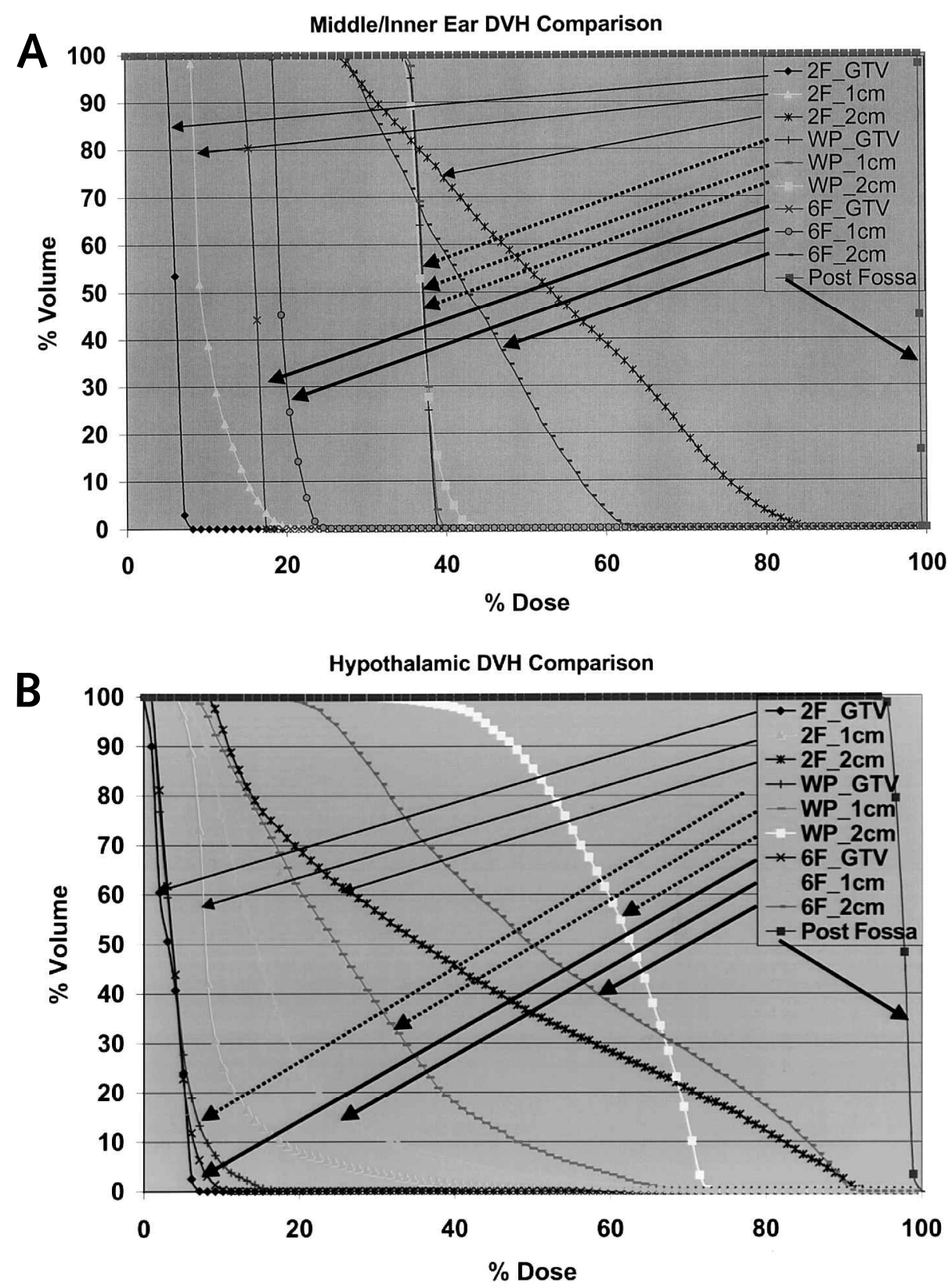

Fig. 2. Dose-volume histograms computed for the (A) middle and inner ear and (B) hypothalamus for each of nine different treatment plans. The nine treatment plans were created from an example case to show the effects of beam orientation and margin on the dose-volume histograms and to compare conventional and conformal methods of posterior fossa irradiation. These plans included parallel-opposed left and right lateral portals with $0.5-, 1.5-$, and $2.5-\mathrm{cm}$ margins on the GTV, paired posterior oblique portals (45 posterior) with $0.5-, 1.5-$, and $2.5-\mathrm{cm}$ margins on the GTV, and six-field beam arrangement (opposed lateral, paired posterior, and superior oblique [45 superior]) with 0.5-, 1.5-, and 2.5-cm margins on the GTV. In addition, we used beam's eye view and standard guidelines to develop a single treatment plan for irradiating the entire posterior fossa. GTV, gross tumor volume; WP, wedge-pair; F, field.

months. Although our current series of patients is small, with a median follow-up of 42 months, no failures have been observed among the patients treated with radiation therapy as a part of their initial management. Given the current requirement of a single cytologic analysis for patients enrolled on cooperative group protocols, it is unlikely that the true incidence of CSF and/or subarachnoid dissemination is reported. Thus, good craniospinal technique appears to control occult dissemination in this group of patients, both intracranially and within the spinal subarachnoid volume.

The focal treatment of tumors in the posterior fossa tests the hypotheses that overall disease control with CRT is equivalent to conventional methods of treatment and reduced treatment volumes result in a reduction in the effects attributable to radiation therapy. Given the intuitive advantages of CRT, it is unlikely that either hypothesis could be tested in a phase III randomized trial. 

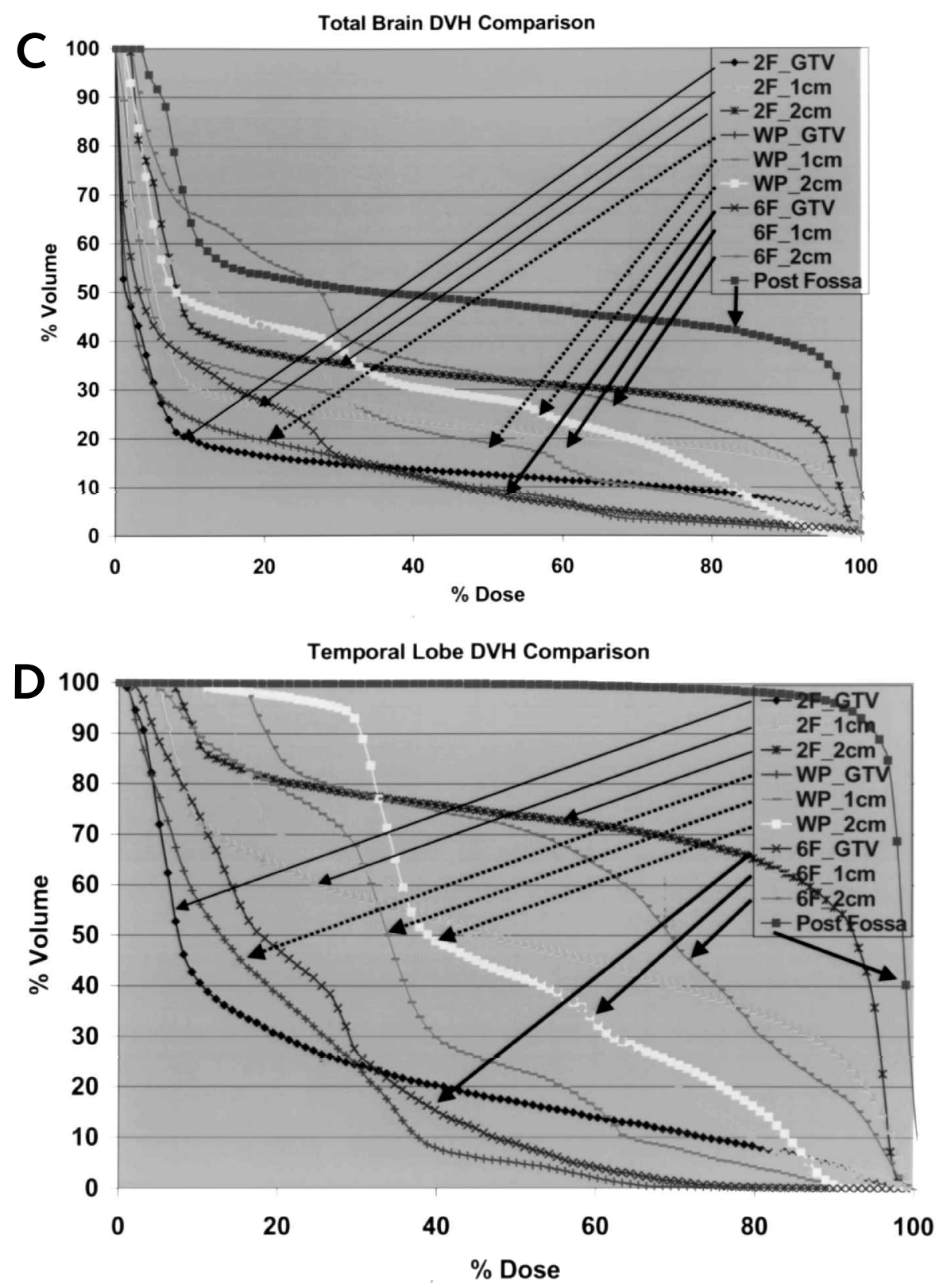

Fig. 2, continued. Dose-volume histograms computed for the (C) total brain and (D) temporal lobe for each of nine different treatment plans. The nine treatment plans were created from an example case to show the effects of beam orientation and margin on the dose-volume histograms and to compare conventional and conformal methods of posterior fossa irradiation. These plans included parallel-opposed left and right lateral portals with $0.5-, 1.5-$, and $2.5-\mathrm{cm}$ margins on the GTV, paired posterior oblique portals (45 posterior) with $0.5-, 1.5-$, and $2.5-\mathrm{cm}$ margins on the GTV, and six-field beam arrangement (opposed lateral, paired posterior, and superior oblique [45 superior]) with $0.5-, 1.5-$, and 2.5 $\mathrm{cm}$ margins on the GTV. In addition, we used beam's eye view and standard guidelines to develop a single treatment plan for irradiating the entire posterior fossa. GTV, gross tumor volume; WP, wedge-pair; F, field.

A phase II trial appears to be more feasible where the patterns of local failure and incidence of radiation-related neurotoxicity could be compared with a control population. In consideration of a phase II trial, where less than the entire posterior fossa would be the targeted volume, the analysis should consider that a portion or all of the posterior fossa subarachnoid volume may receive incidental irradiation, although not at the level of the prescribed dose.

\section{Local Failure in Treatment for Medulloblastoma}

Local failure as a component of treatment failure remains a principal shortcoming in the treatment of medulloblastoma. We reported our long-term results for patients treated with definitive radiation therapy in the CT era (Merchant et al., 1996). With a median follow-up of 8 years, local failure occurred as a component of failure in $55 \%$ of all treatment failures and in $33 \%$ of all failures 


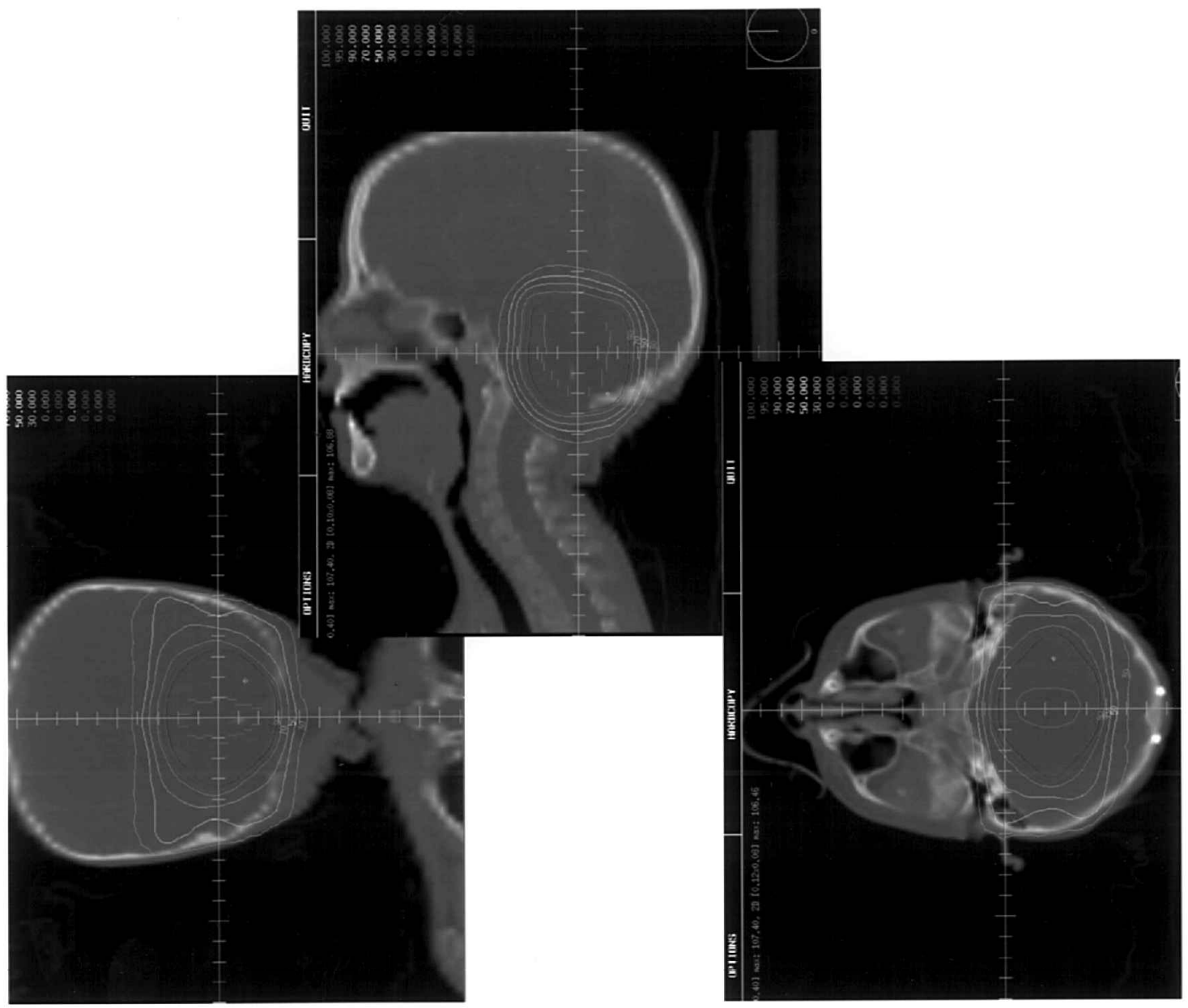

Fig. 3. Axial, coronal, and sagittal dose distributions from six static, individually shaped fields targeting a posterior fossa tumor bed (gross tumor volume) with an anatomically defined clinical tumor volume margin of $1 \mathrm{~cm}$, a $0.5 \mathrm{~cm}$ planning target volume, and a margin of $0.5 \mathrm{~cm}$ for penumbra.

for patients presenting with localized disease. More favorable local control data have been reported (Packer et al., 1994); however, the interpretation of these results should be tempered by the short follow-up for a tumor which is known to recur locally more than 5 years after treatment.

\section{Dose Escalation for Medulloblastoma}

Despite the knowledge that local failure remains an important problem, controversies in the management of medulloblastoma have surrounded the optimal dose of radiation to the craniospinal axis and the benefit of adjuvant chemotherapy. It has not been specifically demonstrated that craniospinal dose or adjuvant chemotherapy influences the pattern of failure at the primary site. The data from infants treated with conventional chemotherapy, given in an effort to delay or obviate the need for radiation therapy, show that the rate of local failure is high enough to warrant the inclusion of radiation therapy in the initial management of these patients (DupuisGirod et al., 1996). With reported long-term results showing substantial primary site failure (Merchant et al.,
1996), some investigators have suggested escalating the dose to the primary site with conventionally or hyperfractionated radiation therapy (Allen et al., 1996). To date, no improvement in local control has been attributed to hyperfractionated radiation therapy, and some investigators remain reluctant to escalate the dose to the posterior fossa primary site fearing excessive toxicity to normal brain, brainstem, or spinal cord. Any risk attributable to dose escalation could be partially offset by using more advanced methods of treatment planning and delivery. Dose escalation also includes the escalation of the total dose with conventional daily fractionation as well as the possibility of boosting areas of residual abnormality with large, single doses of radiation therapy (Graab et al., 1996; Patrice et al., 1995). All of these methods of dose escalation are technically feasible using the techniques described in this report. Seven of the patients treated in this report received 5940 cGy using conventional fractionation (180 cGy/day), and two were treated with $4500 \mathrm{cGy}$ after relapsing following prior radiation therapy to doses in excess of $5000 \mathrm{cGy}$. Similar arguments are made for ependymoma given the high rate of local failure and the poor control with long-term fol- 


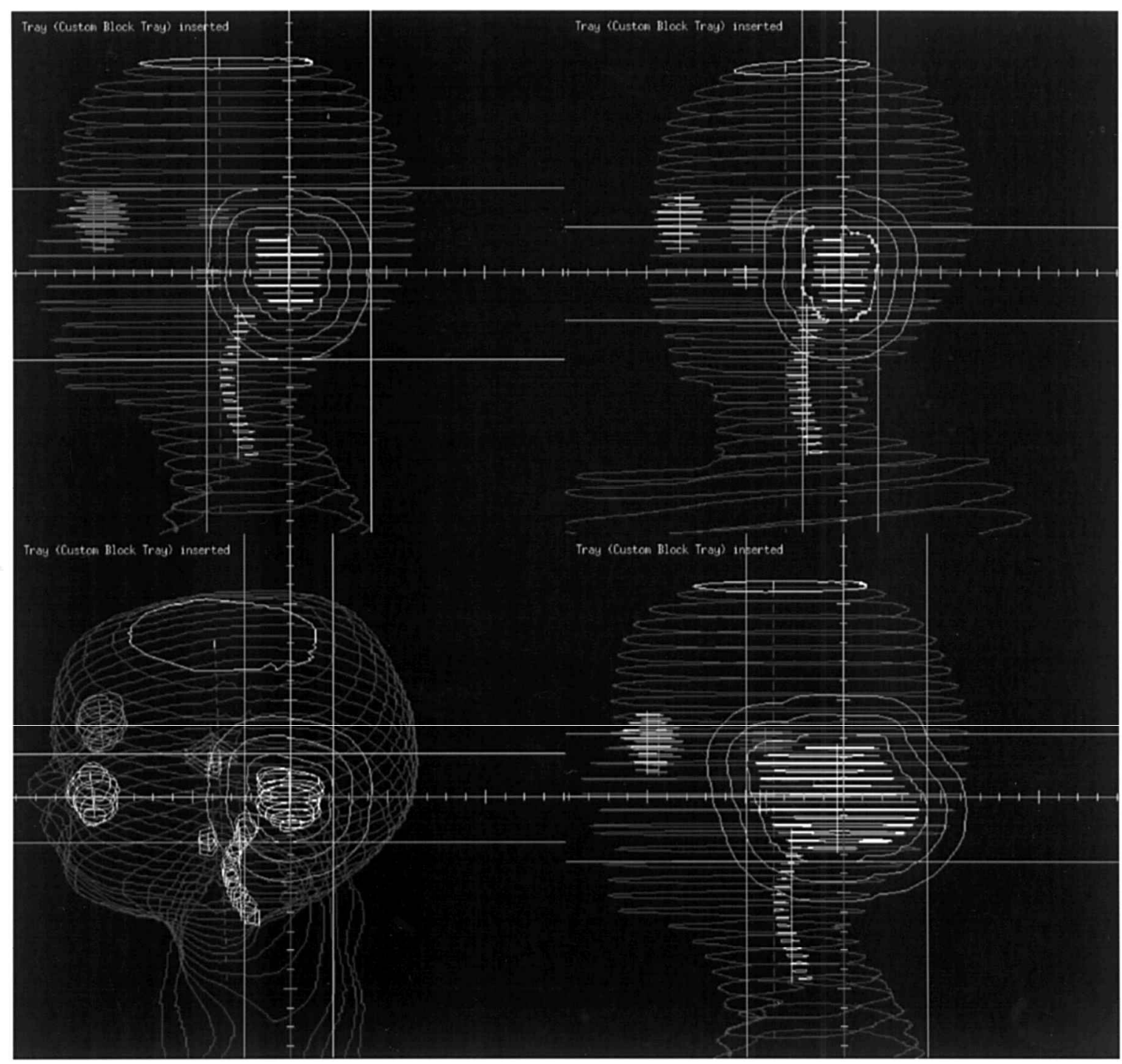

Fig. 4. Lateral (upper right), posterior oblique (upper left), and superior oblique (lower left) portals $(0.5,1.5$, and $2.5 \mathrm{~cm})$ on a midline, IVth ventricle gross tumor volume. Lateral posterior fossa (infratentorial brain) portals using beam's eye view treatment planning. These portals were used to develop the treatment plans that correspond to the dose-volume histogram data presented in Fig. 2 . The structures depicted include the skin surface, the left eye and cochlea, the right eye and cochlea, hypothalamus, spinal cord, gross tumor volume, and beam margins.

low-up (Merchant et al., 1997). Efforts to boost the posterior fossa with high-dose single fraction irradiation appear promising (Aggarwal et al., 1997).

Most studies describing late effects for radiation therapy - whether neuroendocrine, neurocognitive, or audiometric-are confounded by additional factors that also contribute to late toxicity, including the destructive effects of tumor, surgery, and chemotherapy. Objective measures of radiation-related neurotoxicity include neuroendocrine tests, neuropsychometric testing, and neuroimaging. Depending on how the GTV is defined and the margins for the CTV and PTV are chosen, there is a profound difference in the dose delivered to normal tissues using CRT compared with the dose delivered by conventional techniques. The data of this report show that substantial differences in dose to the hypothalamic-pituitary axis, middle and inner ear, supratentorial brain, and cerebellum can be achieved by reducing the treatment volume or altering the beam orientations (Fig. 2). Any measurement specific to the function of the structures spared in the process of delivering conformal treatment would require few patients to corroborate the benefits of CRT.

\section{Reducing Ototoxicity}

This reports shows how CRT can reduce the dose to the middle ear. Radiation therapy alone or in combination with cisplatin, an agent commonly used to treat patients with medulloblastoma and ependymoma, causes sensorineural hearing loss. The true incidence of hearing impairment due to radiation therapy is unknown. There is a general lack of prospective data assessing hearing 
loss in children treated at head and neck and intracranial sites. Evidence is mounting for the long-term survivors of pediatric brain tumors that sensorineural hearing loss is an important radiation-related late effect. Hearing impairment, whether induced by radiation or chemotherapy, leads to delays in neurocognitive development and decreases quality of life. Prospective evaluation of pediatric patients treated at St. Jude Children's Research Hospital ${ }^{3}$ and at the Children's Hospital in New Orleans (Kapoor et al., 1996) revealed that significant hearing loss occurs in approximately $10 \%$ of patients treated with radiation therapy alone $(>15 \mathrm{~dB})$ and in approximately $68-93 \%$ of patients treated with different cumulative doses of cisplatin and radiation therapy $(>30 \mathrm{~dB})$. The median time to significant hearing loss, determined from the analysis of 113 patients evaluated at St. Jude, was 4.1 years. These data fall within the expected range when compared with reports from the adult head and neck literature where the time from radiation therapy to the development of hearing loss ranges from 3 to 6 years (Moretti et al., 1976). It appears that treatment of the auditory apparatus may be superfluous if dosimetric margins are unnecessarily generous or if beam orientations are not considered in the treatment planning process.

\section{The Influence of Radiation Dose and Volume on Intellectual Outcome}

The field of irradiation and the quality of surgery (Hoppe-Hirsch et al., 1995) influence the intellectual outcome for children with malignant tumors of the posterior fossa. Hoppe-Hirsch et al. (1995) showed that, 1-2 years after surgery, $70-80 \%$ of children maintained an IQ greater than 90 when no perioperative complications occurred compared with $20-40 \%$ of patients when a complication did occur. In addition, $90 \%$ of children with ependymoma treated to the posterior fossa alone (field size not described) maintained an IQ greater than 90 at $5-10$ years. These findings compared favorably to the level of IQ performance of children with medulloblastoma who received craniospinal irradiation (25-35 Gy) in addition to a conventional boost to the posterior fossa. In the same series, $20 \%$ of these children at 5 years and $10 \%$ at 10 years maintained an IQ greater than 90 .

These data and others provide little doubt that full-dose neuraxis radiation therapy or whole-brain radiation therapy has a detrimental effect on IQ performance (Mulhern et al., 1992). They support the rationale for cooperative group efforts to test the efficacy of lower doses of craniospinal irradiation for patients with average risk medulloblastoma, especially in the age group of patients for whom differences in IQ outcome are likely to be greatest.
Others have shown that lower doses of craniospinal irradiation (23.4-25 Gy) have a significantly different effect on IQ than does standard-dose radiation therapy to the neuraxis; however, this difference has been confined to patients whose age is less than 9 years (Mulhern et al., 1998). Considering the DVH data of this report, wherein the worse case scenario such as generous posterior fossa portal $50 \%$ (Fig. 2C) of the supratentorial brain receives the prescription dose, it is our hope that the additional sparing of normal brain with the CRT technique will provide a measurable difference in IQ outcome. The benefit of CRT will be most pronounced among medulloblastoma patients who receive the lowest doses of craniospinal irradiation. It follows that neuroendocrine dysfunction occurring after radiation therapy will also benefit from the advancement of these treatment planning techniques. Data concerning endocrine dysfunction occurring after irradiation of the hypothalamic-pituitary unit are limited to the discrete doses used for craniospinal irradiation of medulloblastoma and cranial irradiation for acute lymphoblastic leukemia.

\section{Summary}

We have shown how patients with medulloblastoma may be successfully treated with less than full posterior fossa irradiation using CRT. The success of this approach depends on achieving the same rate of disease control that would be expected if the entire posterior fossa were treated and on reducing the expected rate of radiation-related toxicity. Successful application of CRT techniques will lead to the possibility of including radiation therapy in the primary management of infants with brain tumors and to the possibility of dose escalation in high-risk patients.

While CRT techniques are unlikely to show a benefit in terms of local control and survival, it is hoped that a reduction in dose to normal tissues or critical structures will lead to an improved therapeutic index (reduced side effects). Reductions in toxicity, if objectively validated, might entice investigators to escalate the dose to the primary site and further reduce the chance of local failure that remains an important problem for these patients. In as much as the sagittal MRI can be used to identify the location of the tentorium cerebelli and reduce dose to supratentorial brain in two dimensions, only three-dimensional treatment planning and delivery can optimally avoid or reduce the dose of radiation to normal tissues. There are limitless possibilities with these treatment planning methods, and their use should be individualized. We have attempted to show how the treatment may impact on the distribution of dose and encourage investigators to look at these methods as an alternative to conventional methods of posterior fossa irradiation for medulloblastoma and ependymoma.

\section{References}

Aggarwal, R., Yeung, D., Kumar, P., Muhlbauer, M., and Kun, L.E. (1997) Efficacy and feasibility of stereotactic radiosurgery in the primary management of unfavorable pediatric ependymoma. Radiother. Oncol. 43, 269-273.
Allen, J.C., Donahue, B., DaRosso, R., and Nirenberg, A. (1996) Hyperfractionated craniospinal radiotherapy and adjuvant chemotherapy for children with newly diagnosed medulloblastoma and other primitive 
neuroectodermal tumors. Int. J. Radiat. Oncol. Biol. Phys. 36, $1155-1161$.

Deutsch, M., Thomas, P., Boyett, J., Krischer, J.P., Finlay, J., Kun, L., Hammond, D., and Vietti, T. (1991) Low stage medulloblastoma: A childen's cancer group (CCSG) and pediatric oncology group randomized study of standard vs reduced neuraxis irradiation. Proc. Annu. Meet. Am. Soc. Clin. Oncol. 10, 124. (Abstract)

Dupuis-Girod, S., Hartmann, O., Benhamou, E., Doz, F., Mechinaud, F., Bouffet, E., Coze, C., and Kalifa, C. (1996) Will high dose chemotherapy followed by autologous bone marrow transplantation supplant cranio-spinal irradiation in young children treated for medulloblastoma? J. Neurooncol. 27, 87-98.

Fukunaga-Johnson, N., Lee, J.H., Sandler, H.M., Robertson, P., McNeil, E., and Goldwein, J.W. (1998) Patterns of failure following treatment for medulloblastoma: Is it necessary to treat the entire posterior fossa? Int. J. Radiat. Oncol. Biol. Phys. 42, 143-146.

Graab, P.A., Lunsford, L.D., Albright, A.L., Kondziolka, D., and Flickinger, J.C. (1996) Stereotactic radiosurgery for glial neoplasms of childhood. Neurosurgery 38, 696-702.

Halperin, E.C. (1996) Impact of radiation technique upon the outcome of treatment for medulloblastoma. Int. J. Radiat. Oncol. Biol. Phys. 36, 233-239.

Hoppe-Hirsch, E., Brunet, L., Laroussinie, F., Cinalli, G., Pierre-Kahn, A., Renier, D., Sainte-Rose, C., and Hirsch, J.F. (1995) Intellectual outcome in children with malignant tumors of the posterior fossa: Influence of the field of irradiation and quality of surgery. Childs Nerv. Syst. 11, 340-345.

ICRU (1978). Dose specification for reporting external beam therapy with photons and electrons. ICRU Report 29 (subsequently replaced by ICRU Report 50 dated 1993). Washington, DC: International Commission on Radiation Units and Measurements.

Kapoor, G., Warrier, R.P., Shah, S.J., Tedesco, S., and Yu, L.C. (1996) Cisplatin and cranial irradiation related hearing loss in children. Proc. Am. Soc. Clin. Oncol. 15, 458. (Abstract)
Merchant, T.E., Haida, T., Wang, M.-H., Finlay, J.L., and Liebel, S.A. (1997) Anaplastic ependymoma: Treatment of pediatric patients with or without craniospinal radiation therapy. J. Neurosurg. 86, 943-949.

Merchant, T.E., Wang, M.-H., Haida , T., Lindsley, K.L., Finlay, J., Dunkel, I.J., Rosenblum, M.K., and Leibel, S.A. (1996) Medulloblastoma: Long-term results for patients treated with definitive radiation therapy during the computed tomography era. Int. J. Radiat. Oncol. Biol. Phys. 36, 29-35.

Moretti, J.A. (1976) Sensori-neural hearing loss following radiotherapy to the nasopharynx. Laryngoscope 86, 598-602.

Mulhern, R.K., Hancock, J., Fairclough, D., and Kun, L. (1992) Neuropsychological status of children treated for brain tumors: A critical review and integrative analysis. Med. Pediatr. Oncol. 20, 181-191.

Mulhern, R.K., Kepner, J.L., Thomas, P.R., Armstrong, F.D., Friedman, H.S., and Kun, L.E. (1998) Neuropsychologic functioning of survivors of childhood medulloblastoma randomized to receive conventional or reduceddose craniospinal irradiation: A Pediatric Oncology Group study. J. Clin. Oncol. 16, 1723-1728.

Packer, R.J., Sutton, L.N., Elterman, R., Lange, B., Goldwein, J., Nicholson, H.S., Mulne, L., Boyett, J., D'Angio, G., Wechsler-Jentzsch, K., Reaman, G., Cohen, B.H., Bruce, D.A., Rorke, L.B., Molloy, P., Ryan, J., LaFond, D., Evans, A.E., and Schut, L. (1994) Outcome for children with medulloblastoma treated with radiation and cisplatin, $\mathrm{CCNU}$, and vincristine chemotherapy. J. Neurosurg. 81, 690-698.

Patrice, S.J., Tarbell, N.J., Goumnerova, L.C., Shrieve, D.C., Black, P.M., and Loeffler, J.S. (1995) Results of radiosurgery in the management of recurrent and residual medulloblastoma. Pediatr. Neurosurg. 22, 197-203.

Tomita, T., and McLone, D.G. (1986) Medulloblastoma in childhood: Results of radical resection and low-dose neuraxis radiation therapy. J. Neurosurg. 64, 238-242.

Wara, W.M., Le, Q.-T.X., Sneed, P.K., Larson, D.A., Prados, M.D., Levin, V.A., Edwards, M.S.B., and Weil, M.D. (1994) Pattern of recurrence of medulloblastoma after low-dose craniospinal radiotherapy. Int. J. Radiat. Oncol. Biol. Phys. 30, 551-556. 